\title{
Terapia de rescate en perforación de vía biliar con stent metálico autoexpandible totalmente cubierto posterior a falla en el manejo conservador: reporte de un caso
}

\section{Case Report of Rescue Therapy for Bile Duct Perforation using Fully Covered Self-Expanding Metal Stent after Failure of Conservative Management}

\author{
Germán Tovar F., ${ }^{1}$ Jairo Mendoza S., ${ }^{2}$ Carlos Velandia C., ${ }^{3}$ Carlos López L. ${ }^{4}$
}

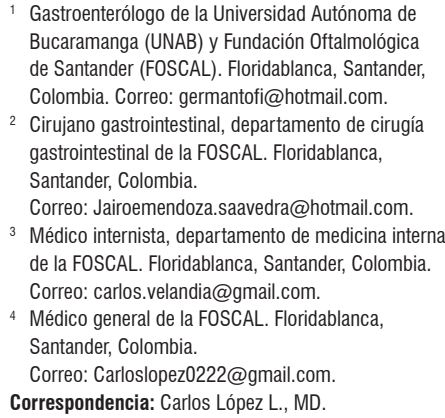

Gastroenterólogo de la Universidad Autónoma de Bucaramanga (UNAB) y Fundación Oftalmológica de Santander (FOSCAL). Floridablanca, Santander, Colombia. Correo: germantofi@hotmail.com.

2 Cirujano gastrointestinal, departamento de cirugía gastrointestinal de la FOSCAL. Floridablanca, Santander, Colombia.

Correo: Jairoemendoza.saavedra@hotmail.com.

3 Médico internista, departamento de medicina interna de la FOSCAL. Floridablanca, Santander, Colombia. Correo: carlos.velandia@gmail.com.

${ }^{4}$ Médico general de la FOSCAL. Floridablanca, Santander, Colombia.

Correo: Carloslopez0222@gmail.com. Correspondencia: Carlos López L., MD.

\begin{abstract}
Resumen
Las complicaciones de la colangiopancreatografía retrógrada endoscópica (CPRE) ocurren entre el 5\% y $10 \%$ de los pacientes, de los cuales menos del $1 \%$ sufre perforaciones. El uso de stents metálicos autoexpandibles totalmente cubiertos para el cierre de perforaciones no complicadas se ha convertido en una estrategia opcional y segura para el manejo inicial de estos pacientes, así como una terapia de rescate para quienes el manejo conservativo ha fallado.

Presentamos el caso de una paciente de 73 años con coledocolitiasis residual que fue sometida a una CPRE terapéutica con esfinterotomía y remoción de los cálculos. 12 horas después de la intervención, consultó al servicio de urgencias donde se confirmó una perforación de la vía biliar tipo II secundaria a esfinterotomía. Ulteriormente, se ofreció un manejo conservador con retiro de la ingesta oral, terapia con inhibidor de la bomba de protones, antibióticos, evaluación quirúrgica y observación médica. Sin embargo, ante la falla del manejo no quirúrgico, se consideró la colocación del stent metálico autoexpandible totalmente cubierto como terapia de rescate con evolución satisfactoria en el postoperatorio y salida 8 días después de la colocación del stent.
\end{abstract}

\section{Palabras clave}

Vía biliar, perforación intestinal, colangiopancreatografía retrógrada endoscópica, postoperatorio.

\begin{abstract}
Complications occur in $5 \%$ to $10 \%$ of patients who undergo endoscopic retrograde cholangiopancreatography (ERCP), but less than $1 \%$ suffer perforations. The use of fully covered self-expanding metal stents (SEMS) to close uncomplicated perforations has become a safe optional strategy for initial management of these patients, as well as for rescue therapy when conservative management has failed.

We present the case of a 73-year-old patient with residual choledocholithiasis who underwent therapeutic ERCP with sphincterotomy and stone removal. Twelve hours after the intervention, the patient returned to the emergency department where a biliary tract type II perforation secondary to sphincterotomy was confirmed. Subsequent conservative management included suspension of oral feeding, administration of proton pump inhibitors and antibiotics, surgical evaluation and medical observation. Failure of non-surgical management led to consideration of placement of a fully covered SEMS as rescue therapy. The patient's postoperative evolution was with satisfactory, and she was discharged eight days after stent placement.
\end{abstract}

\section{Keywords}

Biliary tract, intestinal perforation, endoscopic retrograde cholangiopancreatography, postoperative. 


\section{INTRODUCCIÓN}

La colangiopancreatografía retrógrada endoscópica (CPRE) se introdujo en 1968, desde entonces es un procedimiento apropiado y comúnmente usado para el diagnóstico y manejo de las enfermedades de la vía biliar y pancreática (1). Inicialmente, las intervenciones se restringieron al uso de stents plásticos de pequeño calibre, debido al pequeño diámetro de la vía biliar y pancreática. Sin embargo, desde 1982, el desarrollo de un canal de biopsia de duodenoscopio más grande y nuevas técnicas endoscópicas han hecho posible las intervenciones con stents más grandes (2) y, con esto, la disminución en las complicaciones asociadas como el sangrado post-CPRE $(3,4)$.

Las tasas de complicaciones de la CPRE varían ampliamente en la literatura, la pancreatitis es la complicación más frecuente (5\%-10\%), seguida de hemorragia (1\%-2\%) e infección (1\%-2\%) (5). La mayoría requiere únicamente el manejo conservador en observación médica, con tasas de morbilidad del 5\% al 10\%, y una mortalidad desde el 0,1\% al $1 \%(6,7)$. Sin embargo, la perforación post-CPRE tiene una baja incidencia $(0,5 \%$ y $0,6 \%)$ (5) y alta incidencia en mortalidad ( $7 \%$ al $14 \%$ ), dependiendo de varios factores, una detección temprana y un manejo rápido $(6,8)$.

Las perforaciones ocurren en cualquier punto como el esófago, el estómago y el duodeno durante la endoscopia, en la región periampular asociada con esfinterotomía y en la vía biliar por la instrumentación (9). Los factores de riesgo para la perforación post-CPRE están relacionados con el procedimiento como la esfinterotomía, inyecciones intramurales, dilatación de estenosis, manipulación de la guía, procedimientos prolongados y dificultades durante el examen (10); o también relacionados con el paciente como la anatomía distorsionada por cirugías (p. ej., Billroth II), que confiere un riesgo significante de perforación, que usualmente ocurre en el sitio de la anastomosis o en el muñón eferente (9).

Las perforaciones duodenales post-CPRE se clasifican en 4 tipos de acuerdo con el grado de severidad, la localización anatómica, el mecanismo de la perforación y las implicaciones del manejo según Stapfer y colaboradores (11) (Tabla 1). Una revisión sistemática realizada por Vezakis y colaboradores del 2015 analizó 18 estudios entre el 2000-2014 que incluyeron a 142847 pacientes y encontró una incidencia de perforación duodenal del 0,39\% y, según la clasificación de Stapfer, las perforaciones tipo I fueron del 25\%, las tipo II del $46 \%$ y las tipo III del $22 \%$ con una mortalidad global del 7,8\% (12). El manejo de cada tipo puede ser resumido en 2 categorías: conservador (tipos II, III y IV) y quirúrgico (tipos I y II) (11). La decisión de un acercamiento terapéutico en la perforación tipo II depende de la historia clínica, el examen físico y los hallazgos encontrados en las imágenes de tomografía axial computarizada (TAC) o resonancia magnética $(7,10,11)$ debido a que, luego de una selección cuidadosa de los pacientes, el tratamiento no quirúrgico ha sido exitoso en el 90\% (13). El debate está en decidir cuál manejo no quirúrgico es mejor para el paciente y qué hacer cuando el tratamiento inicial ha fallado, especialmente con las perforaciones tipo II por su altas tasas de mortalidad (12). Según una revisión sistemática realizada por Cirocchi y colaboradores con 303 pacientes, se encontró que el tipo de perforación más frecuente según la clasificación de Stapfer fue tipo II en el $54 \%$ de los casos, de los cuales el $84,2 \%$ requirió únicamente manejo no quirúrgico (14).

En la revisión sistemática realizada por Vezakis y colaboradores, analizaron también 11 estudios sobre el diagnóstico y tratamiento de las perforaciones post-CPRE con una mortalidad global del $9,4 \%$ y encontraron que el $21 \%$ de los pacientes requirió manejo quirúrgico con una mortalidad del $38 \%$ en este grupo de pacientes (12).

La recomendación de los expertos basada en series clínicas es el manejo conservador, con el paso de una sonda nasoduodenal o nasogástrica a drenaje, líquidos endovenosos, antibióticos y, en algunas ocasiones, análogos de la somatostatina; pero sin recomendaciones certeras con respecto a la decisión de un tratamiento agresivo con la colocación de un stent, teniendo en cuenta la preocupación del resultado final y la necesidad de reintervenciones, que es una decisión individualizada para cada paciente (13).

\section{CASO CLÍNICO}

Paciente de 73 años con historia de colecistectomía laparoscópica por colelitiasis. Presentó síntomas clínicos de obstrucción de la vía biliar por coledocolitiasis residual con dilatación secundaria de la vía biliar extrahepática. Se sometió a una CPRE terapéutica con esfinterectomía de la papila mayor y extracción de varios cálculos biliares sin sangrado durante el procedimiento. 12 horas después de la intervención fue admitida al servicio de urgencias por dolor severo en el cuadrante superior derecho que se irradiaba al hombro ipsilateral, asociado con 4 episodios de emesis no hemorrágica. El examen físico mostró una frecuencia cardíaca de 75 latidos por minuto (lpm), con dolor moderado a la palpación del cuadrante superior derecho, sin irritación peritoneal. Los exámenes de laboratorio mostraban un conteo de leucocitos de $11,830 \mathrm{~mm}^{3} \mathrm{y}$ un porcentaje de granulocitos de $91 \%$. Con base en estos hallazgos, se decidió iniciar un manejo conservador que consistió en una terapia con inhibidor de bomba de protones, antibiótico endovenoso y una TAC abdominal con aerobilia y líquido libre en el peritoneo que se extendía desde el borde inferior del hígado hasta el espacio anterior perirrenal derecho (Figura 1). 
Tabla 1. Clasificación de las perforaciones periduodenales post-CPRE (11)

\begin{tabular}{|c|c|c|c|c|}
\hline $\begin{array}{l}\text { Tipo de } \\
\text { perforación }\end{array}$ & Localización anatómica & Mecanismo de perforación & Hallazgos radiológicos & $\begin{array}{l}\text { Grado de } \\
\text { severidad }\end{array}$ \\
\hline I & $\begin{array}{l}\text { Pared medial y lateral del } \\
\text { duodeno }\end{array}$ & Directamente por el endoscopio & $\begin{array}{l}\text { Medio de contraste persistente en el } \\
\text { retroperitoneo, espacio intraperitoneal }\end{array}$ & Moderado/severo \\
\hline$\|$ & $\begin{array}{l}\text { Pared medial del duodeno } 0 \\
\text { perivateriano }\end{array}$ & Esfinterotomía & Fluido peritoneal y retroperitoneal & Moderado \\
\hline III & Distal al ducto biliar & $\begin{array}{l}\text { Instrumentación cerca de la } \\
\text { obstrucción }\end{array}$ & Extravasación mínima de contraste & Moderado \\
\hline IV & Retroperitoneal & $\begin{array}{l}\text { Uso de aire comprimido para } \\
\text { mantener permeabilidad del lumen }\end{array}$ & No visible & Leve \\
\hline
\end{tabular}

Modificado de: Stapfer M et al. Ann Surg. 2000;232(2):191-8.

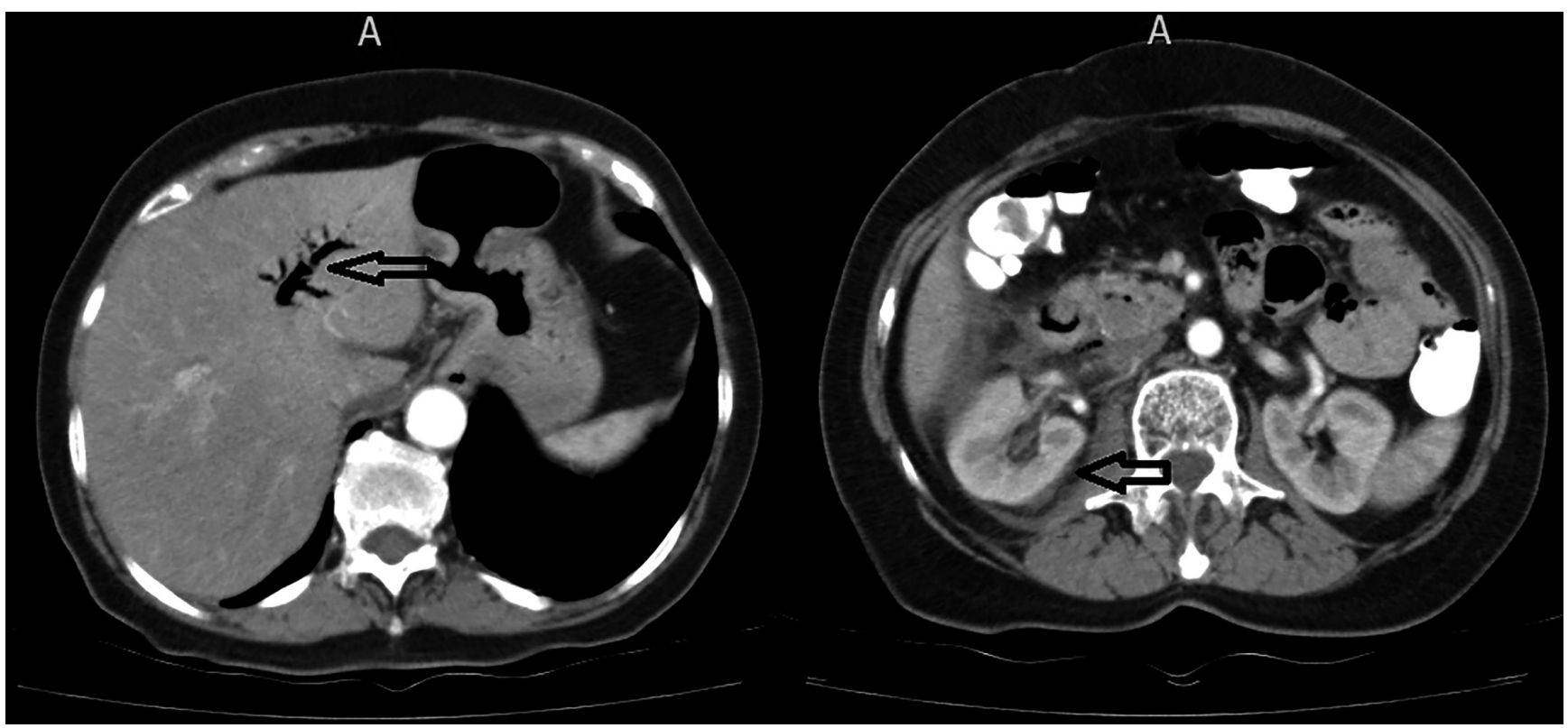

Figura 1. La TAC contrastada de abdomen evidenció aerobilia en el panel izquierdo y en el derecho hubo evidencia de líquido libre en el peritoneo, el hígado y el espacio perirrenal anterior derecho (flechas negras).

El médico del servicio de cirugía gastrointestinal sospechó una perforación duodenal post-CPRE tipo II y decidió continuar el manejo conservador. 48 horas después, la paciente tenía taquicardia, persistencia del dolor abdominal localizado y un recuento de leucocitos en $14,110 \mathrm{~mm}^{3}$ con un porcentaje de neutrófilos del $89 \%$, sin irritación peritoneal. Por estas razones, el cirujano gastrointestinal decidió realizar una CPRE que confirmó la perforación tipo II, con lo que se decidió la colocación de un stent metálico autoexpandible totalmente cubierto (sMAETC) como medida de soporte para el cierre de la perforación, debido a la falla de la terapia medica convencional (Figura 2). Después de la intervención, la evolución clínica de la paciente fue satisfactoria, con ausencia del dolor abdominal, normalización del recuento de leucocitos y una TAC abdominal sin evidencia de salida de material de contraste al retroperitoneo a las 24 horas después del procedimiento.

8 días después de la colocación del sMAETC y la finalización del tratamiento antibiótico, la paciente fue egresada en un estado clínico adecuado. 8 semanas después del procedimiento fue programada para el retiro del stent de manera ambulatoria, sin complicaciones (Figura 3 ).

\section{CONCLUSIONES}

Este reporte describe una perforación post-CPRE tipo II no complicada, la cual falló en el manejo conservativo y requirió la colocación de un sMAETC en la vía biliar como terapia 


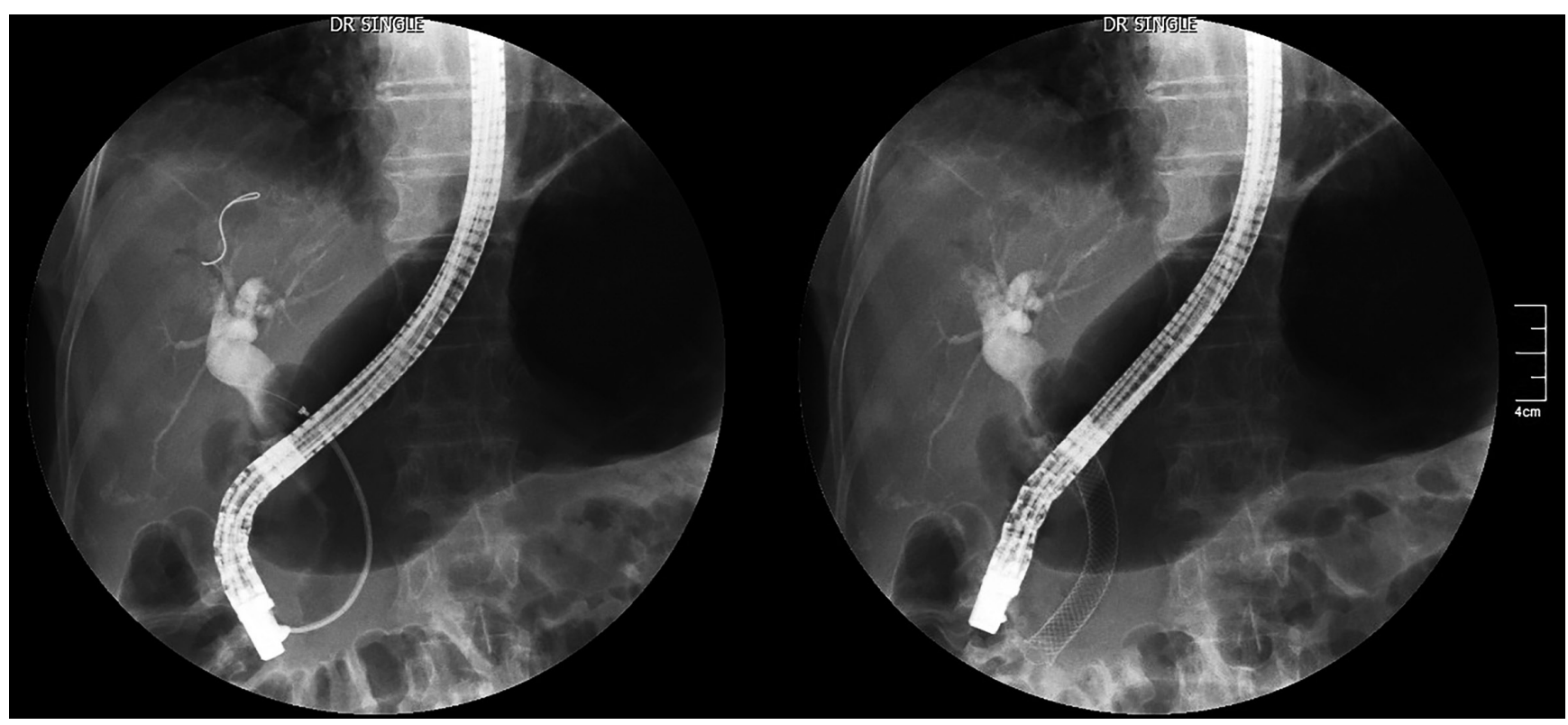

Figura 2. Colangiografía que muestra el sMAETC colocado en la perforación tipo II.

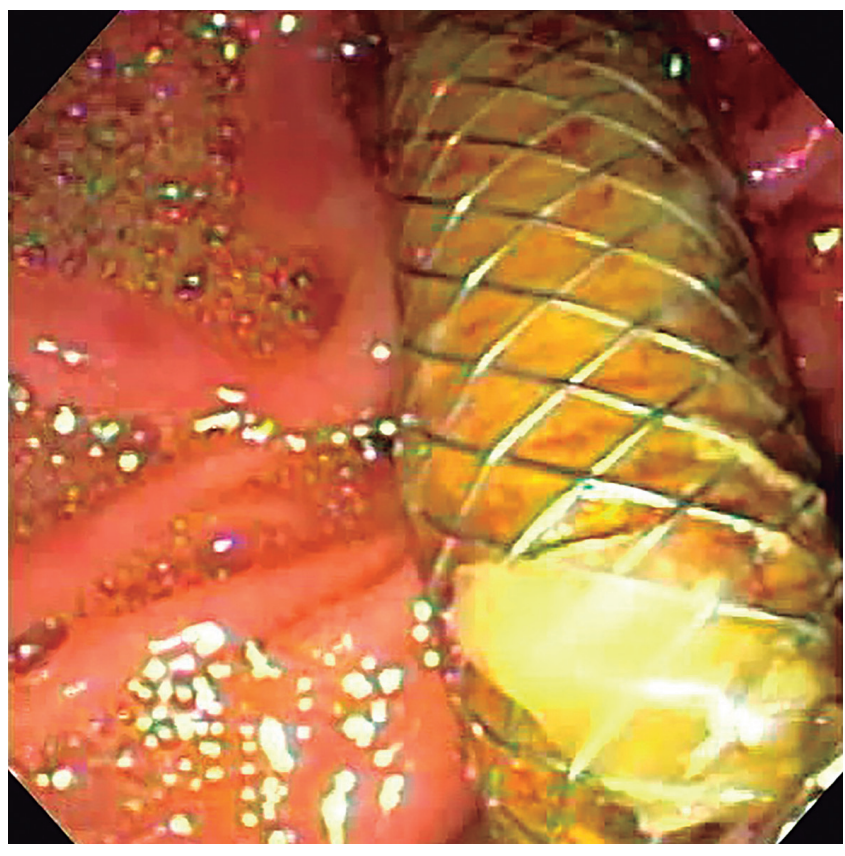

Figura 3. Stent removido 8 semanas después del procedimiento.

de rescate. Aún no hay consenso en las guías de manejo para estas complicaciones, ya que las perforaciones retroperitoneales relacionadas con la endoscopia son raras y la variabilidad en las consecuencias clínicas son amplias (15).

Mantener el tratamiento conservador es el pilar en la mayoría de los casos, incluso con la presencia amplia de aire retroperitoneal en pacientes asintomáticos $(10,13)$. Si se confirma la perforación interna o externa de la vía biliar, el drenaje pancreático puede ser indicado (16). Las contraindicaciones para la aproximación no quirúrgica son la presencia de dolor abdominal con signos de irritación peritoneal, fiebre o signos clínicos de toxicidad, la filtración mayor de medio de contraste, colecciones líquidas y problemas sin resolver, que deben ser resueltos con cirugía, con el reparo o el drenaje de la lesión, especialmente si son adultos mayores $(10,13)$.

Por una parte, con el advenimiento de las nuevas técnicas endoscópicas, las intervenciones mínimamente invasivas con el uso de sMAETC y de endoclips se han reportado para los pacientes con perforaciones tipo I mostrando resultados exitosos $(17,18)$. Por otra parte, Howard y colaboradores reportaron un abordaje innovador y agresivo para las perforaciones tipo II, con el drenaje endoscópico inmediato derivado de la vía biliar, pancreática o el duodeno, proximales a la lesión, usando un stent biliar o un tubo nasobiliar, con drenaje continuo de líquido biliar usando una sonda nasogástrica o nasoduodenal $(16,19)$, con una disminución de la necesidad de intervenciones del $60 \%$ al $10 \%$ (19). Sin embargo, el drenaje biliar inadecuado puede causar infiltración de líquido biliar, que puede llegar a drenar al sitio de la perforación y aumentar la morbilidad (11).

El requerimiento de intervenciones nuevas, seguras y efectivas para el manejo de las complicaciones por perforaciones post-CPRE hizo que desde 2011 se usaran los sMAETC (15), que eran usados en otras condiciones clínicas benignas o neoplásicas de la vía biliar $(20,21)$. El uso en perforaciones tipo II no ha sido ampliamente difundido, solo hay unos pocos reportes de casos, pero con excelentes 
resultados $(15,18,20)$. Una de las preocupaciones acerca de los sMAETC es su seguridad, basados en reportes de complicaciones incluyendo la migración duodenal, úlceras de la vía biliar, coledocolitiasis de novo, estenosis e, incluso, adenomas periampulares $(21,22)$. La evidencia sugiere que la colocación de sMAETC por 30 días o menos es efectiva para resolver fugas de bilis complejas y sangrado postesfinterectomía; la duración del stent requiere variaciones de acuerdo con la condición clínica y la estrategia de un stent temporal está asociada con menor riesgo de complicaciones tempranas o tardías (20).

Aunque las complicaciones secundarias a la CPRE son raras, representan un marcador alto de mortalidad (22); requieren un diagnóstico temprano y un tratamiento multidisciplinario preciso, seguro y efectivo. Este caso alerta sobre la necesidad de una evaluación guiada del abordaje inicial para los pacientes con perforación post-CPRE tipo II no quirúrgica y el manejo de los pacientes con fallo del tratamiento conservativo que no cumplen criterios quirúrgicos, mostrando a los sMAETC como una estrategia innovadora y mínimamente invasiva para el cierre seguro de estas lesiones en pacientes específicos, demostrando el éxito en los desenlaces con el retiro posterior.

\section{Consentimiento}

Se obtuvo el consentimiento informado por escrito del paciente para la publicación de este informe de caso y las imágenes que lo acompañan. Una copia del consentimiento por escrito está disponible para su revisión por el editor de esta revista.

\section{Conflicto de intereses}

Los autores declaran que no tienen conflictos de interés político, personal, religioso, ideológico, académico, intelectual, comercial o cualquier otro.

\section{Agradecimientos}

Agradecemos al Departamento de Radiología que prestó servicios de radiología en nombre de la FOSCAL.

\section{Fuente de apoyo financiero}

No hubo una fuente de apoyo financiero.

\section{REFERENCIAS}

1. McCune WS, Shorb PE, Moscovitz H. Endoscopic cannulation of the ampulla of vater: a preliminary report. Ann Surg.
1968;167(5):752-6. https://doi.org/10.1097/00000658196805000-00013.

2. Huibregtse K, Tytgat GN. Palliative treatment of obstructive jaundice by transpapillary introduction of large bore bile duct endoprosthesis. Gut. 1982;23(5):371-5. https:// doi.org/10.1136/gut.23.5.371.

3. Ferreira LE, Baron TH. Post-sphincterotomy bleeding: who, what, when, and how. Am J Gastroenterol. 2007;102(12):2850-8. https://doi.org/10.1111/j.15720241.2007.01563.x.

4. Valats JC, Funakoshi N, Bauret P, et al. Covered self-expandable biliary stents for the treatment of bleeding after ERCP. Gastrointest Endosc. 2013;78(1):183-7. https://doi. org/10.1016/j.gie.2013.02.035.

5. Loperfido S, Angelini G, Benedetti G, et al. Major early complications from diagnostic and therapeutic ERCP: a prospective multicenter study. Gastrointest Endosc. 1998;48(1):110. https://doi.org/10.1016/S0016-5107(98)70121-X.

6. FreemanML,NelsonDB,ShermanS,etal.Complicationsofendoscopic biliary sphincterotomy. N Engl J Med. 1996;335(13):90918. DOI: 10.1056/NEJM199609263351301.

7. Enns R, Eloubeidi MA, Mergener K, et al. ERCP-related perforations: risk factors and management. Endoscopy. 2002;34(4):293-8. https://doi.org/10.1055/s-2002-23650.

8. Fatima J, Baron TH, Topazian MD, et al. Pancreaticobiliary and duodenal perforations after periampullary endoscopic procedures: diagnosis and management. Arch Surg. 2007;142(5):44854. https://doi.org/10.1001/archsurg.142.5.448.

9. Guda NM, Reddy DN, Kumar A. Complications of ERCP. Indian J Gastroenterol. 2014;33(1):1-9. https://doi. org/10.1007/s12664-013-0383-5.

10. Lee TH, Han JH, Park SH. Endoscopic treatments of endoscopic retrograde cholangiopancreatography-related duodenal perforations. Clin Endosc. 2013;46(5):522-8. https:// doi.org/10.5946/ce.2013.46.5.522.

11. Stapfer M, Selby RR, Stain SC, et al. Management of duodenal perforation after endoscopic retrograde cholangiopancreatography and sphincterotomy. Ann Surg. 2000;232(2):191-8. https://doi.org/10.1097/00000658200008000-00007.

12. Vezakis A, Fragulidis G, Polydorou A. Endoscopic retrograde cholangiopancreatography-related perforations: Diagnosis and management. World J Gastrointest Endosc. 2015;7(14):113541. https://doi.org/10.4253/wjge.v7.i14.1135.

13. Paspatis GA, Dumonceau JM, Barthet $M$, et al. Diagnosis and management of iatrogenic endoscopic perforations: European Society of Gastrointestinal Endoscopy (ESGE) Position Statement. Endoscopy. 2014;46(8):693-711. https://doi.org/10.1055/s-0034-1377531.

14. Cirocchi R, Kelly MD, Griffiths EA, et al. A systematic review of the management and outcome of ERCP related duodenal perforations using a standardized classification system. Surgeon. 2017;15(6):379-87. https://doi.org/10.1016/j. surge.2017.05.004.

15. Vezakis A, Fragulidis G, Nastos C, et al. Closure of a persistent sphincterotomy-related duodenal perforation by pla- 
cement of a covered self-expandable metallic biliary stent. World J Gastroenterol. 2011;17(40):4539-41. https://doi. org/10.3748/wjg.v17.i40.4539.

16. Cotton PB, Lehman G, Vennes J, et al. Endoscopic sphincterotomy complications and their management: an attempt at consensus. Gastrointest Endosc. 1991;37(3):383-93. https://doi.org/10.1016/S0016-5107(91)70740-2.

17. Small AJ, Petersen BT, Baron TH. Closure of a duodenal stent-induced perforation by endoscopic stent removal and covered self-expandable metal stent placement (with video). Gastrointest Endosc. 2007;66(5):1063-5. https:// doi.org/10.1016/j.gie.2007.03.1082.

18. Baron TH, Wong Kee Song LM, Zielinski MD, et al. A comprehensive approach to the management of acute endoscopic perforations (with videos). Gastrointest Endosc. 2012;76(4):838-59. https://doi.org/10.1016/j. gie.2012.04.476.
19. Thomas J. Howard. Letters To The Editor. Ann Surg. 2001;234(1):132-3.

20. Canena J, Liberato M, Horta D, et al. Short-term stenting using fully covered self-expandable metal stents for treatment of refractory biliary leaks, postsphincterotomy bleeding, and perforations. Surg Endosc. 2013;27(1):313-24. https://doi. org/10.1007/s00464-012-2368-3.

21. Kahaleh M, Behm B, Clarke BW, et al. Temporary placement of covered self-expandable metal stents in benign biliary strictures: a new paradigm? (with video). Gastrointest Endosc. 2008;67(3):446-54. https://doi.org/10.1016/j. gie.2007.06.057.

22. Sandha GS, Bourke MJ, Haber GB, et al. Endoscopic therapy for bile leak based on a new classification: results in 207 patients. Gastrointest Endosc. 2004;60(4):567-74. https:// doi.org/10.1016/S0016-5107(04)01892-9. 